\title{
The Role of Local Business Employees and Community Members in The HIV Risk Environment of Female Sex Workers in An Urban Setting: Associations Between Negative Interactions and Inconsistent Condom Use
}

\section{Susan G Sherman ( $\nabla$ ssherman@jhu.edu )}

\section{Catherine Tomko}

Johns Hopkins University Bloomberg School of Public Health https://orcid.org/0000-0003-2622-5518

Bradley E Silberzahn

University of Texas at Austin

\section{Rebecca Hamilton White}

Johns Hopkins University Bloomberg School of Public Health

\section{Danielle Friedman Nestadt}

Johns Hopkins University Bloomberg School of Public Health

\section{Emily Clouse}

Johns Hopkins University Bloomberg School of Public Health

\section{Katherine Haney}

Johns Hopkins University Bloomberg School of Public Health

Noya Galai

Johns Hopkins University Bloomberg School of Public Health

\section{Research article}

Keywords: female sex work, risk environment, condom use, HIV

Posted Date: July 27th, 2020

DOI: https://doi.org/10.21203/rs.3.rs-44248/v1

License: (c) (i) This work is licensed under a Creative Commons Attribution 4.0 International License. Read Full License

Version of Record: A version of this preprint was published at BMC Public Health on December 1st, 2021. See the published version at https://doi.org/10.1186/s12889-021-12293-4. 


\section{Abstract}

Background: The role of business employees and community members in the HIV risk environment of female sex workers (FSW) is underexplored, despite sex work often located in commercial and residential urban areas. We explored the effect of negative interactions between business employees and community members inconsistent condom use with clients.

Methods: This study uses baseline data from the EMERALD study, a structural intervention with FSW. We recruited a sample of 361 FSW in Baltimore, Maryland using targeted sampling techniques in ten zones throughout the city. Participants were recruited between September 2017 and January 2019 and completed a survey, HIV rapid testing, and self-administered gonorrhea and chlamydia testing. The outcome, inconsistent condom use, was defined as not reporting "always" using condoms with paying clients. Poisson regressions with robust variance were used to model the effect of business employee and/or community member interactions on inconsistent condom use.

Results: Over half (54\%) the sample was between 18-40 years old, 44\% Black or another race, and experienced a range of structural vulnerabilities such as housing instability and food insecurity. Forty-four percent of the sample reported inconsistent condom use with clients. FSW reported being reported to the police weekly or daily for selling drugs ( $14 \%$ by employees, $17 \%$ by community), for selling sex ( $19 \%$ by employees, $21 \%$ by community), and experiencing weekly or daily verbal or physical threats ( $18 \%$ by employees, $24 \%$ by community). In multivariable models, being reported to the police for selling sex weekly or daily by community members (vs. never, aOR $=1.42,95 \% \mathrm{Cl}=1.08,1.86$ ) and business owners (vs. never, aOR $=1.36,95 \% \mathrm{Cl}=1.05,1.76$ ) increased odds of inconsistent condom use, as did monthly verbal or physical threats by community members (vs. never, aOR $=1.43,95 \% \mathrm{Cl}=1.08,1.91$ ).

Conclusions: Results show that both actors play important roles in FSWs' HIV risk environment. Businesses and community members are important targets for holistic HIV prevention interventions among FSW in communities where they coexist in close proximity.

\section{Background}

A growing body of evidence has identified a complexity of factors that impact the HIV risk of female sex workers (FSWs) in their work environments. (1-5) Over the last decade, complex physical, social, economic, and policy environmental features have been articulated and examined in their shaping of HIV transmission and related behaviors. (6-9) Yet little is known about the role of business owners and community members in shaping FSW HIV risk. Given their omnipresence in communities where street-based FSW work, their potential influence merits examination.

Rhodes' risk environment framework provides a useful framework as it is a theoretical approach that shifts the attribution of risk from that of the individual to the broader society, structures, and contexts in which they live and work. $(10,11)$ The framework suggests that behaviors and associated risks are positioned and influenced by the context in which they occur. These contexts can produce, heighten, or minimize individuals' risks. Accordingly, health is tied to social conditions and structural factors rather than individual actions alone. $(11,12)$ This framework is rooted in a line of research that posits the dynamic interplay of individuals and their environments, which are characterized by complex systems that are dynamic and interactive. (13-15)

A diversity of environmental features of sex work have been implicated in drug and sexual risk for female sex workers (FSW), with police driving some of the most significant and egregious risks. $(4,6,16-18)$ In the context of illegality, police are a key feature of FSW's HIV risk environment, with their impact intensified on FSWs who work on the street. (4, $18,19)$ Studies have documented significant associations between egregious police behaviors (e.g., sexual coercion, extortion) and HIV/STI infection. (20-23) In the U.S., urban renaissances characterized by redevelopment and 
gentrification has changed the landscape of many cities with new development and introduced new neighborhood residents and local businesses. (24-26) Gentrification is often accompanied by elevated police activity to reduce crime in neighborhoods, resulting in moving drug and sex work markets and creating barriers to health care and other outreach services. $(27,28)$ Further, community's complaints and police responses can further "spatially marginalize" FSW, often moving them to sell sex in unfamiliar locations. (25) The physical isolation of the practice of street-based FSW has been associated with violence, unsafe sex; sharing drug use equipment) and lack of access to health services. $(29,30)$ Yet little is known about the nature of FSWs' engagement with key players in the environments in which they work.

Additionally, police verbal harassment or sexual and physical assault have been associated with an increased risk of inconsistent condom use with clients and client violence. $(4,21,31-33)$ Police attention to sex work in neighborhoods largely is in response from community complaints. Yet this initial step in the cascade of harms from police and clients, has been omitted from studies of contextual risk among this population. Recourse for police and client violence and other egregious behaviors is eclipsed by the illegal nature of sex work, functionally limiting women's negotiating power that can often result in condom coercion. (32-38) The impact of these and other environmental features are amplified in the context of limited economic opportunities and drug addiction. (39-41)

In urban areas in the U.S., the street-based sex work scene is located in residential or commercial areas where businesses or community residents regularly interact with FSW. $(26,42)$ Residents of these areas cite moralized concerns about the reputation of the neighborhood or potential increase in violent crime as reasons for anti-sex work sentiments, though these sentiments are largely owing to stereotyping rather than experience. $(24,26,43)$ This study aims to fill this gap in our understanding of the complexity of the risk environment's impacts on FSWs' health, we examine community members' and police's impact on sexual risk, namely inconsistent condom use.

\section{Methods}

Data for this analysis are derived from the baseline EMERALD study, an evaluation of a HIV prevention, community-level intervention among street-based FSW in Baltimore, Maryland. The intervention includes a full-service drop-in Center that provides medical, addiction, mental health, social service, and legal services as well as drop-in services (e.g., showers, lockers, laundry, food, a place to rest, therapeutic art groups, yoga). A detailed description of the study protocol and intervention have been published previously. (44) Briefly, EMERALD is a prospective comparative study that used a targeted sampling technique previously employed by a cohort study of FSW in Baltimore along with updated geospatial analyses of potential sex work activity indicators (e.g. 911 calls for prostitution, arrest data) to develop an updated spatial sampling frame for FSW recruitment. (45) Between September 2017 and February 2019, study staff recruited FSW from 10 street recruitment locations, six of which were in the intervention area and four were in the comparison area.

EMERALD eligibility criteria included: 1) aged 18 or older; 2) cis-gender woman; 3) sold or traded oral, vaginal, or anal sex "for money or things like food, drugs, or favors (past 3 months);" 4) picked up clients 3 or more times (past 3 months); and 5) willing to provide contact information for follow up visits. Eligible participants provided written informed consent, a 50-minute Audio Computer-Assisted Self-Interview (ACASI) survey consisting of demographics, sex work history, drug use, and psychosocial measures. Participants also completed a rapid oral HIV test (OraQuick) and provided a self-administered vaginal swab which was sent to the Baltimore City Department of Health for gonorrhea and chlamydia testing. The study was approved by [blinded for review].

\section{Outcome}


Participants were asked separately about frequency of recent (past week) condom use ("from the beginning to end of penetration") during vaginal or anal sex with new and regular clients. Responses included: never, rarely, sometimes, most of the time, always, and did not have [vaginal/anal] sex. Inconsistent condom use (ICU) was defined as reporting any frequency other than "always" during vaginal or anal sex with new or regular clients.

\section{Independent Variables}

Demographics (e.g., age, ethnicity) and structural vulnerability context factors including homelessness, experiencing hunger ("because there was not enough food to eat") at least weekly vs. less than weekly, financial dependence on someone, having financial dependent(s), and sources of income. We measured symptoms of post-traumatic stress disorder (PTSD) with the PTSD Patient Checklist (PCL-5) (scores $\geq 33$ indicated clinically significant PTSD symptoms) and depression with the Patient Health Questionnaire-9 (scores > 10 indicated presence of depressive symptoms) and recent (past 6 months) substance use include injecting any drug and using crack or powdered cocaine or heroin via any method. $(46,47)$

Sex work history variables included age started selling sex, frequency of sex exchange in past 6 months (daily vs. else), location(s) where women picked up new clients in the past 6 months, number of clients per week (split at the median, 15 vs. $\geq 6$ ), and if "someone takes a cut" of their sex work earnings. We also measured FSW responses to police practices in the past 6 months including rushing negotiations with clients due to police in the area, avoiding carrying condoms, or moving to an unfamiliar area to work to avoid police. Recent client-perpetrated physical or sexual violence was measured using an adapted version of the Revised Conflict Tactic Scale. (48)

\section{Community Member and Business Employees Interactions}

Participants reported the frequency, if ever, of types of interactions with business employees and community members including: a) reporting them to the police for using drugs; b) asking them to leave an area in which they are selling sex;

c) reporting them to police for selling sex; d) verbally or physically threatening them. The eight response options ranged from never to every day and were collapsed into three categories: never, monthly, and weekly or daily. One binary yes/no question assessed whether women "rushed negotiations in the last 6 months because of [business employees/community members] in the area." All questions were asked separately of business employees and community members.

\section{Statistical Analysis}

The sample size is 361 women. The analysis excluded 24 participants, 15 of whom did not answer questions related to community member and business employees questions and 9 who not report engaging in vaginal and anal sex with new and regular clients. We used Pearson's $\chi^{2}$ tests to evaluate differences in business employees or community members interactions, and other covariates by ICU. We used Poisson regressions with robust variance estimates for univariate regressions to determine unadjusted odds ratios of ICU by employee and community interactions and other covariates. Covariates at the $\mathrm{p}<0.20$ level were considered in subsequent multivariable models. We then used Poisson regressions with robust variance estimates for three separate multivariable models: Model $A$ includes only business employee variables and covariates, Model B includes only community member variables and covariates, and Model C includes both business employee and community member variables and covariates. We used Akaike information criterion (AIC), Bayesian information criterion (BIC), and variance inflation factors to select the best fitting models and retained any covariates $p<0.15$ in the final models. We fit models $A, B$, and $C$ separately, though the same covariates emerged as significant in each. Significance was set at alpha $=0.05$. All analyses were conducted in Stata/SE 15.1 (College Station, TX). 


\section{Results}

The cohort description with demographic, structural vulnerability, substance use, mental health, and sex work histories are reported in Table 1, stratified by reporting inconsistent condom use. Over two-thirds of the sample (65\%) was between 18-39 years old and $57 \%$ was white (Table 1). A majority (67\%) reported experiencing recent homelessness, $59 \%$ reported experiencing hunger at least weekly, and about half reporting clinically-significant PTSD (49\%) and depressive (46\%) symptoms. Most women (59\%) sold sex for longer than 10 years, and over half (52\%) sold sex daily. Gonorrhea or chlamydia prevalence at baseline was $15 \%$ and $21 \%$, respectively, and HIV prevalence was $6 \%$. 
Table 1

Sample characteristics stratified by inconsistent condom use with clients among $n=361$ female sex workers (FSW) in Baltimore, Maryland

$\begin{array}{llll}\text { No Inconsistent Condom } & \text { Inconsistent Condom } & \text { Total }(n & P \\ \text { Use }(n=201) & \text { Use }(n=160) & =361)\end{array}$

\section{Demographics}

Age

$18-29$

$45(22.4)$

$39(24.4)$

$84(23.3)$

$30-39$

86 (42.8)

57 (35.6)

143

(39.6)

40-49

52 (25.9)

42 (26.3)

94 (26.0)

$50+$

18 (9.0)

22 (13.8)

40 (11.1)

Education: 3 level

$<$ HS grad

92 (45.8)

73 (45.6)

0.92

HS grad/GED

52 (25.9)

57 (28.4)

39 (24.4)

48 (30.0)

165

(45.7)

Some col +

$57(28.4)$

91 (25.2)

105

(29.1)

Race

White

116 (57.7)

86 (53.8)

0.45

Non-white

85 (42.3)

74 (46.3)

50 (31.4)

$93(26.1)$

0.04

\section{Economic Context}

Homeless, past 6 months

$134(66.7)$

105 (65.6)

239

(66.2)

0.32

202

(56.0)

Experienced hunger...

Less than weekly

$82(40.8)$

57 (35.6)

0.32

At least weekly

119 (59.2)

103 (64.4)

(38.5)

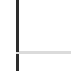

Depend on someone financially

$146(72.6)$

127 (79.4)

222

(61.5)

Others depend on you financially

95 (47.3)

79 (49.4)

273

(75.6)

Sex work only source of income

53 (26.4)

$41(25.6)$

174

(48.2)

Mental Health

$\star<3 \%$ data missing 


\begin{tabular}{|c|c|c|c|c|}
\hline & $\begin{array}{l}\text { No Inconsistent Condom } \\
\text { Use }(n=201)\end{array}$ & $\begin{array}{l}\text { Inconsistent Condom } \\
\text { Use }(n=160)\end{array}$ & $\begin{array}{l}\text { Total }(\mathrm{n} \\
=361)\end{array}$ & $\mathbf{P}$ \\
\hline $\begin{array}{l}\text { Clinically-significant PTSD } \\
\text { symptoms* }\end{array}$ & $96(49.0)$ & $93(58.5)$ & $\begin{array}{l}189 \\
(53.2)\end{array}$ & 0.07 \\
\hline $\begin{array}{l}\text { Clinically-significant depressive } \\
\text { symptoms* }\end{array}$ & $92(46.2)$ & $74(46.8)$ & $\begin{array}{l}166 \\
(46.5)\end{array}$ & 0.91 \\
\hline \multicolumn{5}{|l|}{ Substance Use } \\
\hline Used crack/cocaine, past 6 months & $178(88.6)$ & $138(86.3)$ & $\begin{array}{l}316 \\
(87.5)\end{array}$ & 0.51 \\
\hline Used heroin, past 6 months & $166(82.6)$ & $123(76.9)$ & $\begin{array}{l}289 \\
(80.1)\end{array}$ & 0.18 \\
\hline Injected drugs, past 6 months & $119(59.2)$ & $92(57.5)$ & $\begin{array}{l}211 \\
(58.4)\end{array}$ & 0.74 \\
\hline \multicolumn{5}{|l|}{ Sex Work History \& Characteristics } \\
\hline Time in sex work & & & & 0.99 \\
\hline$\leq 10$ years & $83(41.3)$ & $66(41.3)$ & $\begin{array}{l}149 \\
(41.3)\end{array}$ & \\
\hline$>10$ years & $118(58.7)$ & $94(58.8)$ & $\begin{array}{l}212 \\
(58.7)\end{array}$ & \\
\hline Age started sex work* & & & & 0.41 \\
\hline$<18$ & $38(19.0)$ & $36(22.8)$ & $74(20.7)$ & \\
\hline $18-30$ years & $119(59.5)$ & $83(52.5)$ & $\begin{array}{l}202 \\
(56.4)\end{array}$ & \\
\hline $30+$ years & $43(21.5)$ & $39(24.7)$ & $82(22.9)$ & \\
\hline Sold sex daily & $104(51.7)$ & $86(53.8)$ & $\begin{array}{l}190 \\
(52.6)\end{array}$ & 0.7 \\
\hline Found new clients on the street* & $157(78.1)$ & $141(88.7)$ & $\begin{array}{l}298 \\
(82.8)\end{array}$ & 0.01 \\
\hline \multicolumn{5}{|l|}{ Client per week } \\
\hline 1 to 5 & $101(50.3)$ & $54(33.8)$ & $\begin{array}{l}155 \\
(42.9)\end{array}$ & 0.002 \\
\hline $6+$ & $100(49.8)$ & $106(66.3)$ & $\begin{array}{l}206 \\
(57.1)\end{array}$ & \\
\hline $\begin{array}{l}\text { Someone takes a cut of your } \\
\text { earnings }\end{array}$ & $26(12.9)$ & $25(15.6)$ & $51(14.1)$ & 0.47 \\
\hline Gonorrhea positive & $30(15.4)$ & $23(14.8)$ & $53(15.1)$ & 0.89 \\
\hline Chlamydia positive & $41(20.8)$ & $23(14.9)$ & $64(18.2)$ & 0.16 \\
\hline \multicolumn{5}{|l|}{ Violence } \\
\hline $\begin{array}{l}\text { Forced or pressured sex from } \\
\text { clients, past } 6 \text { months* }^{\star}\end{array}$ & $62(30.8)$ & $74(46.5)$ & $\begin{array}{l}136 \\
(37.8)\end{array}$ & 0.002 \\
\hline$\star<3 \%$ data missing & & & & \\
\hline
\end{tabular}




\begin{tabular}{|c|c|c|c|c|}
\hline & $\begin{array}{l}\text { No Inconsistent Condom } \\
\text { Use }(n=201)\end{array}$ & $\begin{array}{l}\text { Inconsistent Condom } \\
\text { Use }(n=160)\end{array}$ & $\begin{array}{l}\text { Total }(\mathrm{n} \\
=361)\end{array}$ & $\mathbf{P}$ \\
\hline $\begin{array}{l}\text { Client physical violence, past } 6 \\
\text { months }\end{array}$ & $54(26.9)$ & $66(41.3)$ & $\begin{array}{l}120 \\
(33.2)\end{array}$ & 0.002 \\
\hline $\begin{array}{l}\text { Client sexual violence, past } 6 \\
\text { months* }\end{array}$ & $40(19.9)$ & 53 (33.3) & $93(25.8)$ & 0.004 \\
\hline \multicolumn{5}{|l|}{ Response to Police Practices } \\
\hline Rushed negotiations, past 6 months & $85(42.7)$ & $77(48.4)$ & $\begin{array}{l}162 \\
(45.3)\end{array}$ & 0.28 \\
\hline $\begin{array}{l}\text { Avoided carrying condoms, past } 6 \\
\text { months }\end{array}$ & $20(10.0)$ & $35(21.9)$ & $55(15.3)$ & 0.002 \\
\hline $\begin{array}{l}\text { Moved to an unfamiliar area to } \\
\text { work, past } 6 \text { months }\end{array}$ & $29(14.7)$ & $35(21.9)$ & $64(17.9)$ & 0.08 \\
\hline$\star<3 \%$ data missing & & & & \\
\hline
\end{tabular}

Prevalence of ICU with clients was $44 \%$. Women who used condoms inconsistently were significantly more likely to have recently been arrested, found new clients on the street, have six or more clients per week, and reported having experienced recent forced or pressured sex, physical violence, and sexual violence from clients. They were also more significantly likely to avoid carrying condoms due to police in the area.

Table 2 displays frequencies of business employee or community member interactions stratified by inconsistent condom use. Twenty-eight percent of women said they were asked to leave the area in which they sold sex on a daily or weekly basis, $18 \%$ were verbally or physically threatened by employees daily or weekly, and $31 \%$ rushed negotiations with clients because of business employees in the area daily or weekly. Seventeen percent of women said they were reported to the police for using drugs on a daily or weekly basis, $27 \%$ reported being asked to leave the area in which they sold sex daily or weekly, and $36 \%$ rushed negotiations with clients because of community members in the area on a daily or weekly basis. Women reporting ICU were more likely to be reported to the police by business employees daily or weekly for using drugs $(p=0.02)$ or for selling sex $(p=0.03)$ compared to women who reported consistent condom use. Women reporting ICU were more likely to be reported to the police by community members daily or weekly for selling sex $(p=0.04)$ and to be verbally or physically threatened by community members daily or weekly $(p=0.03)$ compared to women who reported consistent condom use. 
Table 2

Frequencies of business employee or community member interactions stratified by inconsistent condom use among $\mathrm{n}$ $=361$ female sex workers (FSW) in Baltimore, Maryland

\begin{tabular}{|c|c|c|c|c|c|c|c|c|}
\hline & $\begin{array}{l}\text { No } \\
\text { Inconsistent } \\
\text { Condom } \\
\text { Use (n= } \\
201)\end{array}$ & $\begin{array}{l}\text { Inconsistent } \\
\text { Condom } \\
\text { Use }(n= \\
160)\end{array}$ & $\begin{array}{l}\text { Total } \\
(n= \\
361)\end{array}$ & $\mathbf{P}$ & $\begin{array}{l}\text { No } \\
\text { Inconsistent } \\
\text { Condom } \\
\text { Use }(n= \\
201)\end{array}$ & $\begin{array}{l}\text { Inconsistent } \\
\text { Condom } \\
\text { Use }(n= \\
160)\end{array}$ & $\begin{array}{l}\text { Total } \\
(\mathrm{n}= \\
361)\end{array}$ & $\mathbf{P}$ \\
\hline & \multicolumn{3}{|c|}{ Business Employees } & \multicolumn{3}{|c|}{ Community Members } & & \\
\hline \multicolumn{9}{|c|}{ Frequency of ___ reporting you to the police for using drugs } \\
\hline Never & $156(80.0)$ & $106(67.5)$ & $\begin{array}{l}262 \\
(74.4)\end{array}$ & $0.02^{*}$ & $139(70.2)$ & $96(60.8)$ & $\begin{array}{l}235 \\
(66.0)\end{array}$ & $0.17 *$ \\
\hline Monthly & $16(8.2)$ & $24(15.3)$ & $\begin{array}{l}40 \\
(11.4)\end{array}$ & & $30(15.2)$ & $31(19.6)$ & $\begin{array}{l}61 \\
(17.1)\end{array}$ & \\
\hline Weekly/Daily & $23(11.8)$ & $27(17.2)$ & $\begin{array}{l}50 \\
(14.2)\end{array}$ & & $29(14.5)$ & $31(19.6)$ & $\begin{array}{l}60 \\
(16.9)\end{array}$ & \\
\hline \multicolumn{9}{|c|}{ Frequency of __ asking you to leave the area that you are selling sex } \\
\hline Never & 105 (53.3) & $71(44.7)$ & $\begin{array}{l}176 \\
(49.4)\end{array}$ & $0.22^{\star}$ & $103(51.2)$ & $65(40.6)$ & $\begin{array}{l}168 \\
(46.5)\end{array}$ & 0.13 \\
\hline Monthly & $43(21.8)$ & $37(23.3)$ & $\begin{array}{l}80 \\
(22.5)\end{array}$ & & $48(23.9)$ & $46(28.8)$ & $\begin{array}{l}94 \\
(26.0)\end{array}$ & \\
\hline Weekly/Daily & $49(24.9)$ & $51(32.1)$ & $\begin{array}{l}100 \\
(28.1)\end{array}$ & & $50(24.9)$ & $49(30.6)$ & $\begin{array}{l}99 \\
(27.4)\end{array}$ & \\
\hline \multicolumn{9}{|c|}{ Frequency of ___ reporting you to the police for selling sex } \\
\hline Never & $139(69.5)$ & $90(56.3)$ & $\begin{array}{l}229 \\
(63.6)\end{array}$ & $0.03^{*}$ & $125(62.2)$ & $80(50.0)$ & $\begin{array}{l}205 \\
(56.8)\end{array}$ & 0.04 \\
\hline Monthly & $34(17.0)$ & $30(18.8)$ & $\begin{array}{l}64 \\
(17.8)\end{array}$ & & $42(20.9)$ & $37(23.1)$ & $\begin{array}{l}79 \\
(21.9)\end{array}$ & \\
\hline Weekly/Daily & $27(13.5)$ & $40(25.0)$ & $\begin{array}{l}67 \\
(18.6)\end{array}$ & & $34(16.9)$ & $43(26.9)$ & $\begin{array}{l}77 \\
(21.3)\end{array}$ & \\
\hline \multicolumn{9}{|c|}{ Frequency of __ verbally or physically threatening you } \\
\hline Never & $140(69.7)$ & $103(64.4)$ & $\begin{array}{l}243 \\
(67.3)\end{array}$ & 0.36 & $132(65.7)$ & $87(54.4)$ & $\begin{array}{l}218 \\
(60.7)\end{array}$ & 0.03 \\
\hline Monthly & $30(14.9)$ & $23(14.4)$ & $\begin{array}{l}53 \\
(14.7)\end{array}$ & & $23(11.4)$ & $33(20.6)$ & $\begin{array}{l}56 \\
(15.5)\end{array}$ & \\
\hline Weekly/Daily & $31(15.4)$ & $34(21.3)$ & $\begin{array}{l}65 \\
(18.1)\end{array}$ & & $46(23.9)$ & $40(25.0)$ & $\begin{array}{l}86 \\
(23.8)\end{array}$ & \\
\hline \multicolumn{9}{|c|}{ Rushed negotiations with clients because of ___ in the area } \\
\hline Ever & $56(27.9)$ & $57(35.6)$ & $\begin{array}{l}113 \\
(31.3)\end{array}$ & 0.19 & $64(31.8)$ & $66(41.3)$ & $\begin{array}{l}130 \\
(36.0)\end{array}$ & 0.17 \\
\hline
\end{tabular}


Model A examined the relationship between interactions with business employees and ICU, controlling for key psychosocial and sex work characteristics (Table 3). Compared to women who never experience business employees reporting them to police for selling sex, being reported to the police weekly or daily was associated with greater odds of ICU $(\mathrm{aRR}=1.36,95 \% \mathrm{Cl}=1.05,1.76)$. In the presence of other variables, there were significant elevated odds of ICU in association with: finding new clients on the street (adjusted risk ratio [aRR] $=1.58,95 \%$ confidence interval $[\mathrm{Cl}]=1.06$, $2.35)$, experiencing forced or pressured sex from clients $(a R R=1.30,95 \% \mathrm{Cl}=1.04,1.63)$, having six or more clients per week (compared to $1-5$, aRR $=1.37,95 \% \mathrm{Cl}=1.07,1.77)$, and avoided carrying condoms because of police $(\mathrm{aRR}=1.33$, $95 \% \mathrm{Cl}=1.02,1.72)$. 
Table 3

Bivariate and multivariable Poisson models with robust variance modeling inconsistent condom use among $n=361$ female sex workers (FSW) in Baltimore, Maryland

\begin{tabular}{|c|c|c|c|c|c|c|c|c|}
\hline & \multicolumn{2}{|c|}{$\begin{array}{l}\text { Unadjusted } \\
\text { Models }\end{array}$} & \multicolumn{2}{|c|}{ Model A } & \multicolumn{2}{|c|}{ Model B } & \multicolumn{2}{|c|}{ Model C } \\
\hline & $\begin{array}{l}\mathrm{RR}(95 \% \\
\mathrm{Cl})\end{array}$ & $\mathbf{p}$ & aRR & $\mathbf{p}$ & $\begin{array}{l}\text { aRR } \\
(95 \% \\
\mathrm{Cl})\end{array}$ & $\mathrm{p}$ & $\begin{array}{l}\text { aRR } \\
(95 \% \\
\text { Cl) }\end{array}$ & $\mathrm{p}$ \\
\hline \multicolumn{9}{|l|}{ Business Employee Variables } \\
\hline \multicolumn{9}{|l|}{$\begin{array}{l}\text { Frequency of reporting you to the police } \\
\text { for using drugs (ref = never) }\end{array}$} \\
\hline Monthly & $\begin{array}{l}1.48 \\
(1.11 \\
1.99)\end{array}$ & 0.01 & - & - & & & - & - \\
\hline Weekly/Daily & $\begin{array}{l}1.35 \\
(0.99 \\
1.79)\end{array}$ & 0.06 & - & - & & & - & - \\
\hline \multicolumn{9}{|l|}{$\begin{array}{l}\text { Frequency of reporting you to the police } \\
\text { for selling sex (ref = never) }\end{array}$} \\
\hline Monthly & $\begin{array}{l}1.19 \\
(0.88 \\
1.62)\end{array}$ & 0.26 & $\begin{array}{l}1.06 \\
(0.78 \\
1.43)\end{array}$ & 0.71 & & & $\begin{array}{l}1.04 \\
(0.75 \\
1.42)\end{array}$ & 0.83 \\
\hline Weekly/Daily & $\begin{array}{l}1.52 \\
(1.18 \\
1.96)\end{array}$ & 0.001 & $\begin{array}{l}1.36 \\
(1.05 \\
1.76)\end{array}$ & 0.02 & & & $\begin{array}{l}1.33 \\
(0.99 \\
1.79)\end{array}$ & 0.06 \\
\hline $\begin{array}{l}\text { Ever rushed negotiations with clients } \\
\text { because of business owners } \\
\text { (ref = never) }\end{array}$ & $\begin{array}{l}1.22 \\
(0.97 \\
1.55)\end{array}$ & 0.09 & - & - & & & - & - \\
\hline \multicolumn{9}{|l|}{ Community Member Variables } \\
\hline $\begin{array}{l}\text { Frequency of reporting you to the police } \\
\text { for selling sex (ref }=\text { never) }\end{array}$ & & & & & - & - & - & - \\
\hline Monthly & $\begin{array}{l}1.20 \\
(0.90 \\
1.61)\end{array}$ & 0.22 & & & $\begin{array}{l}1.01 \\
(0.75 \\
1.36)\end{array}$ & 0.96 & $\begin{array}{l}0.99 \\
(0.72 \\
1.35)\end{array}$ & 0.93 \\
\hline Weekly/Daily & $\begin{array}{l}1.45 \\
(1.12 \\
1.88)\end{array}$ & 0.01 & & & $\begin{array}{l}1.42 \\
(1.08 \\
1.86)\end{array}$ & 0.01 & $\begin{array}{l}1.26 \\
(0.93 \\
1.70)\end{array}$ & 0.14 \\
\hline \multicolumn{9}{|l|}{$\begin{array}{l}\text { Frequency of verbally or physically } \\
\text { threatening you (ref = never) }\end{array}$} \\
\hline Monthly & $\begin{array}{l}1.48 \\
(1.13 \\
1.95)\end{array}$ & 0.01 & & & $\begin{array}{l}1.43 \\
(1.08 \\
1.91)\end{array}$ & 0.01 & $\begin{array}{l}1.44 \\
(1.08 \\
1.90)\end{array}$ & 0.01 \\
\hline Weekly/Daily & $\begin{array}{l}1.17 \\
(0.89 \\
1.55)\end{array}$ & 0.27 & & & $\begin{array}{l}0.88 \\
(0.66 \\
1.18)\end{array}$ & 0.40 & $\begin{array}{l}0.84 \\
(0.62 \\
1.14)\end{array}$ & 0.27 \\
\hline
\end{tabular}




\begin{tabular}{|c|c|c|c|c|c|c|c|c|}
\hline \multirow[b]{2}{*}{$\begin{array}{l}\text { Ever rushed negotiations with clients } \\
\text { because of community members } \\
\text { (ref = never) }\end{array}$} & \multicolumn{2}{|c|}{$\begin{array}{l}\text { Unadjusted } \\
\text { Models }\end{array}$} & \multirow[t]{2}{*}{ Model A } & & \multicolumn{2}{|c|}{ Model B } & \multicolumn{2}{|c|}{ Model C } \\
\hline & $\begin{array}{l}1.25 \\
(0.99 \\
1.58)\end{array}$ & 0.06 & & & - & - & - & - \\
\hline \multicolumn{9}{|l|}{ Contextual Characteristics } \\
\hline Arrested, past 6 months $(r e f=$ no $)$ & & 0.03 & & - & & - & & - \\
\hline Yes & $\begin{array}{l}1.30 \\
(1.03, \\
1.65)\end{array}$ & & - & & - & & - & \\
\hline PTSD symptoms (ref = no) & & 0.07 & & - & & - & & - \\
\hline Yes & $\begin{array}{l}1.24 \\
(0.98 \\
1.57)\end{array}$ & & - & & - & & - & \\
\hline $\begin{array}{l}\text { Found new clients on the street } \\
(\mathrm{ref}=\mathrm{no})\end{array}$ & & 0.02 & & 0.03 & & 0.05 & & 0.02 \\
\hline Yes & $\begin{array}{l}1.63 \\
(1.08 \\
2.45)\end{array}$ & & $\begin{array}{l}1.58 \\
(1.06, \\
2.35)\end{array}$ & & $\begin{array}{l}1.58 \\
(1.07 \\
2.34)\end{array}$ & & $\begin{array}{l}1.58 \\
(1.07 \\
2.34)\end{array}$ & \\
\hline $\begin{array}{l}\text { Client forced or pressured sex } \\
(\text { ref }=\text { no) }\end{array}$ & & 0.002 & & 0.02 & & 0.05 & & 0.05 \\
\hline Yes & $\begin{array}{l}1.44 \\
(1.14 \\
1.80)\end{array}$ & & $\begin{array}{l}1.30 \\
(1.04, \\
1.63)\end{array}$ & & $\begin{array}{l}1.25 \\
(1.00 \\
1.58)\end{array}$ & & $\begin{array}{l}1.26 \\
(1.00 \\
1.58)\end{array}$ & \\
\hline Client per week $(r e f=1-5)$ & & & & 0.01 & & 0.01 & & 0.01 \\
\hline $6+$ & & & $\begin{array}{l}1.37 \\
(1.07 \\
1.77)\end{array}$ & & $\begin{array}{l}1.39 \\
(1.08 \\
1.78)\end{array}$ & & $\begin{array}{l}1.38 \\
(1.08, \\
1.77)\end{array}$ & \\
\hline $\begin{array}{l}\text { Avoided carrying condoms because } \\
\text { of police }(\mathrm{ref}=\mathrm{no})\end{array}$ & & $\begin{array}{l}< \\
0.001\end{array}$ & & 0.03 & & 0.01 & & 0.01 \\
\hline Yes & $\begin{array}{l}1.55 \\
(1.22, \\
1.98)\end{array}$ & & $\begin{array}{l}1.33 \\
(1.02 \\
1.72)\end{array}$ & & $\begin{array}{l}1.41 \\
(1.10 \\
1.81)\end{array}$ & & $\begin{array}{l}1.37 \\
(1.06, \\
1.76)\end{array}$ & \\
\hline $\begin{array}{l}\text { Moved to unfamiliar area because of } \\
\text { police }(\mathrm{ref}=\mathrm{no})\end{array}$ & & 0.06 & & - & & - & & - \\
\hline Yes & $\begin{array}{l}1.29 \\
(0.99, \\
1.67)\end{array}$ & & - & & - & & - & \\
\hline
\end{tabular}

\section{Multivariable Model with Community Members (Model B)}

Model B examined the relationship between interactions with community members and ICU, controlling for key psychosocial and sex work characteristics. Compared to women who never experienced verbal or physical threats from community members, experiencing threats monthly was associated with greater odds of ICU (aRR $=1.43,95 \% \mathrm{Cl}=1.08$, 1.91), though experiencing threats weekly or daily was not significantly associated with ICU. Compared to women who 
never experience community members reporting them to police for selling sex, being reported to the police weekly or daily was associated with greater odds of $\mathrm{ICU}(\mathrm{aRR}=1.42,95 \% \mathrm{Cl}=1.05,1.76)$. Additionally, there were significant elevated odds of ICU in association with: finding new clients on the street $(\mathrm{aRR}=1.58,95 \% \mathrm{Cl}=1.07,2.34)$, experiencing forced or pressured sex from clients $(\mathrm{aRR}=1.25,95 \% \mathrm{Cl}=1.00,1.58)$, having six or more clients per week (compared to $1-5, a R R=1.39,95 \% \mathrm{Cl}=1.08,1.78)$, and avoided carrying condoms because of police $(\mathrm{aRR}=1.41,95 \% \mathrm{Cl}=1.10,1.81)$.

\section{Multivariable Model with Business Employees and Community Members (Model C)}

Model $\mathrm{C}$ examined the relationship between interactions with business employees and community members, controlling for key psychosocial and sex work characteristics, which were similarly significant in this model as models A and B. Further, when including both business employee and community member interaction in the model, ICU was only associated with experiencing verbal or physical threats from community members on a monthly basis compared to never experiencing threats ( $\mathrm{aRR}=1.44,95 \% \mathrm{Cl}=1.08,1.90)$.

\section{Discussion}

This study examines the negative impact of businesses and community members on sexual risk among a sample of sex workers. This is one of the first studies to identify these groups as social features of FSWs' occupational risk environment. We found a significant association between negative interactions with both business and community members on inconsistent condom use with clients. Findings indicate the frequency and impact of these exchanges on women's HIV and STI risk beyond salient structural, mental health, and sex work-related factors.

The frequency of interactions with businesses and community members was high. In separate models, frequently (at least weekly) being reported to the police by both business employees and community members was significantly associated with inconsistent client use in the presence of key demographic and sex work characteristics. Threats of verbal or physical abuse by community members (at least monthly) were associated with inconsistent condom use with clients. Lastly, when business and community interactions were in the same model controlling for key relevant demographic and sex work characteristics, monthly verbal or physical threats from community members maintained significance. Overall, women reported more frequent interactions with community members compared to business owners, likely owing to their being in the neighborhood more often than business employees at night when women are working. Although not directly related to unprotected sex, these types of engagements with community members and businesses likely creates hostile work environments for FSW.

FSW possess little recourse for physical or verbal threats or calls to the police given the criminalization of sex work. Engaging with the police, whether threats (e.g., verbal harassment) or actions (e.g., arrest, sexual violence) is a number of harmful outcomes. $(16,33,49)$ In the current study, the impact of police was reflected in the significant association between client inconsistent condom use with both avoiding carrying condoms and moving to unfamiliar areas in all three adjusted models, both attributed to the police. An earlier study of FSW in Baltimore found associations between abusive policing practices and client-perpetrated violence, which, in turn, has been found to increase risk for inconsistent condom use.(4) Sex work decriminalization would afford many protections to women including their ability to organize and negotiate around where they conduct their business.

The study sheds light on the role of these social aspects of FSW's HIV risk environment, complimenting existing literature that examines the nature and impact of the built environment on sexual and drug related risk among this population. $(9,17)$ Similar to street-based FSW in Baltimore and elsewhere in the US, reported rates of illicit drug use are high. $(16,50)$ Overlapping drug and sex markets likely impact communities synergistically, fueling animosity toward drug-using FSW and exacerbating their interactions with them.(17) Further, community members' and businesses' frustrations over the impact of the sex and drug economies on their neighborhoods could result in disproportionate responses on FSW compared to male who use drugs.

Page 13/18 
The current sample was characterized by several structural vulnerabilities, including low prevalence of education and high prevalence of homelessness, hunger, and arrest. Structural vulnerability refers to the position of an individual within social structures and the impact of those structures such as laws (e.g., illegality of prostitution or drug use), policing, and access to resources such as housing and employment.(12) The totality of these factors can render women less able to weather the impact of frequent negative interactions with businesses and community members. The occurrence of sex work in a given neighborhood can be perceived by residents and businesses as a driver for devaluating property values, placing disproportionate blame on these women for external economic, urban planning, and broader drug market trends. $(43,51)$ This misplaced anger may exacerbate FSW stigma, resulting in further marginalization and isolation of women in moving them to work in more concentrated, hidden, and dangerous spaces, thereby increasing their exposure to violence and risk. $(7,17,52)$

A recent study examining business and community members opinions toward a proposed overdose prevention site (OPS) in their neighborhood found high OPS acceptability with some demographic differences despite media reports about residents' frustrations with public drug use in their neighborhood. (53) A similar survey among businesses and community members would provide an understanding their attitudes and behaviors toward FSW that could be used to informed tailored interventions to reduce FSW stigma and potentially support partial FSW decriminalization in indoor spaces, reducing the footprint of sex work in their neighborhood.

The current study is characterized by several limitations. It is important to remember that these results are not reflective of all FSW. Our sample is recruited from the street and primarily, but not exclusively, find clients on the street; they are also characterized by a number of structural vulnerabilities. Further, sex work is illegal in the United States and findings with respect to threats of police involvement are not likely generalizable to decriminalized sex work environments. Second, we collected data using ACASI software on tablet computers, which can provide more privacy than intervieweradministered surveys but limits the ability to probe unclear responses. Finally, data is self-reported and may be subject to desirability bias especially the outcome of client condom use.

\section{Conclusions}

In summary, the results from our study highlight the importance of a number of aspects of the HIV risk environment in its direct and indirect negative impact on street-based FSW. Businesses and community members are salient environmental features of FSWs' workspace, that should be included in holistic HIV prevention and occupational safety interventions for FSWs. The prevalence of negative interactions with these populations including being reported to the police, physically or verbally threatened, or being reported to the police creates both a hostile work and impacts women's sexual risk profile. The impact of sex work criminalization and stigma is far reaching, and in this case, tacitly giving license for maltreatment of sex workers by others who are perceived or actually impacted by their occupational location. Police more likely to protect business and residential interests than that of FSW in any of these encounters, with the tool of arrest not only failing to reduce sex work but also generating a number of harms. Future research is necessitated to understand the complexity of interactions between FSW and these actors. Further, research with businesses and community would provide insights to understand their perspectives and point to levers of change to both engage them in HIV prevention and occupational safety interventions.

\section{Abbreviations}

FSW: female sex worker

HIV: human immunodeficiency virus 
STI: sexually transmitted infection

ACASI: audio computer assisted self-interview

AIC: Akaike information criterion

BIC: Bayesian information criterion

PTSD: post-traumatic stress disorder

ICU: inconsistent condom use

RR: risk ratio

Cl: confidence interval

OPS: overdose prevention site

\section{Declarations}

Ethics approval and consent to participate: Participants provided written consent prior to joining the study. All study activities were approved by the Johns Hopkins Bloomberg School of Public Health Institutional Review Board.

Availability of data and materials: The datasets analyzed during the current study are not publicly available due participant confidentiality but are available from the corresponding author on reasonable request.

Competing interests: The authors declare that they have no conflict of interest.

Funding: National Institute on Drug Abuse (R01DA041243); National Institute of Mental Health (F31MH118817); and Johns Hopkins University Center for AIDS Research, a National Institutes of Health funded program (P30AI094189). The funders had no role in the design of the study, collection, analysis, or interpretation of data, or writing the manuscript.

Authors' contributions: SGS secured study funding, conceptualized the research question, interpreted the data, and drafted and substantively revised the manuscript. CT conceptualized the research question, analyzed the data, interpreted the data, and drafted and substantively revised the manuscript. BES acquired the data and substantively revised the manuscript. RHW and DFN interpreted the data and substantively revised the manuscript. EC and $\mathrm{KH}$ acquired data and substantively revised the manuscript. NG contributed to the conception and design of the manuscript, interpreted the data, and substantively revised the manuscript. All authors approved the submitted version and agreed to be accountable for their contributions.

Acknowledgements: We thank the EMERALD field staff for their many hours in the field. We also thank the EMERALD participants for this time and insights.

\section{References}

1. Goldenberg SM, Duff P, Krusi A. Work environments and HIV prevention: a qualitative review and meta-synthesis of sex worker narratives. BMC public health. 2015; 15(1):1241.

2. Goldenberg SM, Jiménez TR, Brouwer KC, Miranda SM, Silverman JG. Influence of indoor work environments on health, safety, and human rights among migrant sex workers at the Guatemala-Mexico Border: a call for occupational health and safety interventions. BMC international health and human rights. 2018; 18(1):9.

Page 15/18 
3. Duff P, Sou J, Chapman J, et al. Poor working conditions and work stress among Canadian sex workers. Occupational Medicine. 2017; 67(7):515-21.

4. [author, blinded for review].

5. McBride B, Goldenberg SM, Murphy A, et al. Third parties (venue owners, managers, security, etc.) and access to occupational health and safety among sex workers in a Canadian setting: 2010-2016. American journal of public health. 2019; 109(5):792-8.

6. [author, blinded for review].

7. Shannon K, Kerr T, Allinott S, Chettiar J, Shoveller J, Tyndall MW. Social and structural violence and power relations in mitigating HIV risk of drug-using women in survival sex work. Soc Sci Med. 2008; 66(4):911-21. doi:10.1016/j.socscimed.2007.11.008

8. Shannon K, Strathdee SA, Goldenberg SM, et al. Global epidemiology of HIV among female sex workers: influence of structural determinants. Lancet. 2015; 385(9962):55-71. doi:10.1016/S0140-6736(14)60931-4

9. Blanchard JF, Aral SO. Emergent properties and structural patterns in sexually transmitted infection and HIV research. Sexually Transmitted Infections. 2010; 86(Suppl 3):iii4-iii9.

10. Rhodes T, Wagner K, Strathdee SA, Shannon K, Davidson P, Bourgois P. Structural violence and structural vulnerability within the risk environment: theoretical and methodological perspectives for a social epidemiology of HIV risk among injection drug users and sex workers. Rethinking social epidemiology: Springer; 2012. p. 205-30.

11. Rhodes T. Risk environments and drug harms: a social science for harm reduction approach. Elsevier; 2009.

12. Quesada J, Hart LK, Bourgois P. Structural vulnerability and health: Latino migrant laborers in the United States. Med Anthropol. 2011; 30(4):339-62. doi:10.1080/01459740.2011.576725

13. Diez Roux AV. Integrating social and biologic factors in health research: a systems view. Ann Epidemiol. 2007; 17(7):569-74. doi:10.1016/j.annepidem.2007.03.001

14. Binson D, Woods WJ. A theoretical approach to bathhouse environments. Journal of Homosexuality. 2003; 44(34):23-31.

15. Moos RH, Lemke S. Evaluating residential facilities: The multiphasic environmental assessment procedure: Sage Publications, Inc; 1996.

16. [author, blinded for review].

17. Deering KN, Amin A, Shoveller J, et al. A systematic review of the correlates of violence against sex workers. Am J Public Health. 2014; 104(5):e42-54. doi:10.2105/AJPH.2014.301909

18. [author, blinded for review].

19. Platt L, Grenfell P, Meiksin R, et al. Associations between sex work laws and sex workers' health: A systematic review and meta-analysis of quantitative and qualitative studies. PLoS medicine. 2018; 15(12):e1002680.

20. [author, blinded for review].

21. Erausquin JT, Reed E, Blankenship KM. Change over Time in Police Interactions and HIV Risk Behavior Among Female Sex Workers in Andhra Pradesh, India. AIDS Behav. 2015; 19(6):1108-15. doi:10.1007/s10461-014-0926-5

22. Decker MR, Crago AL, Chu SK, et al. Human rights violations against sex workers: burden and effect on HIV. Lancet. 2015; 385(9963):186-99. doi:10.1016/S0140-6736(14)60800-X

23. [author, blinded for review]. 
24. Mathieu L. Neighbors' anxieties against prostitutes' fears: ambivalence and repression in the policing of street prostitution in France. Emotion, Space and Society. 2011; 4(2):113-20.

25. Hubbard P. Community action and the displacement of street prostitution: evidence from British cities. Geoforum. 1998; 29(3):269-86.

26. Hubbard P, Boydell S, Crofts P, Prior J, Searle G. Noxious neighbours? Interrogating the impacts of sex premises in residential areas. Environment and Planning A. 2013; 45(1):126-41.

27. Goldenberg SM, Amram O, Braschel M, Moreheart S, Shannon K. Urban gentrification and declining access to $\mathrm{HIV/STI}$, sexual health, and outreach services amongst women sex workers between 2010-2014: Results of a community-based longitudinal cohort. Health \& Place. 2020:102288.

28. Linton SL, Cooper HL, Kelley ME, et al. Cross-sectional association between ZIP code-level gentrification and homelessness among a large community-based sample of people who inject drugs in 19 US cities. BMJ open. 2017; 7(6):e013823.

29. Lazarus L, Chettiar J, Deering K, Nabess R, Shannon K. Risky health environments: women sex workers' struggles to find safe, secure and non-exploitative housing in Canada's poorest postal code. Social Science \& Medicine. 2011; 73(11):1600-7.

30. Rhodes T, Simić M, Baroš S, Platt L, Žikić B. Police violence and sexual risk among female and transvestite sex workers in Serbia: qualitative study. Bmj. 2008; 337:a811.

31. Decker MR, Lyons C, Billong SC, et al. Gender-based violence against female sex workers in Cameroon: prevalence and associations with sexual HIV risk and access to health services and justice. Sex Transm Infect. 2016;

92(8):599-604. doi:10.1136/sextrans-2015-052463

32. [author, blinded for review].

33. Duff P, Birungi J, Dobrer S, Akello M, Muzaaya G, Shannon K. Social and structural factors increase inconsistent condom use by sex workers' one-time and regular clients in Northern Uganda. AIDS Care. 2018; 30(6):751-9. doi:10.1080/09540121.2017.1394966

34. Wirtz AL, Schwartz S, Ketende S, et al. Sexual violence, condom negotiation, and condom use in the context of sex work: results from two West African countries. J Acquir Immune Defic Syndr. 2015; 68 Suppl 2:S171-9.

doi:10.1097/QAI.0000000000000451

35. Shannon K, Strathdee SA, Shoveller J, Rusch M, Kerr T, Tyndall MW. Structural and environmental barriers to condom use negotiation with clients among female sex workers: implications for HIV-prevention strategies and policy. Am J Public Health. 2009; 99(4):659-65. doi:10.2105/AJPH.2007.129858

36. Lang DL, Salazar LF, DiClemente RJ, Markosyan K. Gender based violence as a risk factor for HIV-associated risk behaviors among female sex workers in Armenia. AIDS and Behavior. 2013; 17(2):551-8.

37. Wechsberg WM, Luseno WK, Lam WK. Violence against substance-abusing South African sex workers: intersection with culture and HIV risk. AIDS Care. 2005; 17 Suppl 1:S55-64. doi:10.1080/09540120500120419

38. Beattie TS, Bhattacharjee P, Ramesh BM, et al. Violence against female sex workers in Karnataka state, south India: impact on health, and reductions in violence following an intervention program. BMC Public Health. 2010; 10:476. doi:10.1186/1471-2458-10-476

39. Deuba K, Anderson S, Ekstrom AM, et al. Micro-level social and structural factors act synergistically to increase HIV risk among Nepalese female sex workers. Int J Infect Dis. 2016; 49:100-6. doi:10.1016/j.ijid.2016.06.007

40. Pauw I, Brener L. 'You are just whores-you can't be raped': barriers to safer sex practices among women street sex workers in Cape Town. Culture, health \& sexuality. 2003; 5(6):465-81. 
41. Aral SO, St Lawrence JS. The ecology of sex work and drug use in Saratov Oblast, Russia. Sex Transm Dis. 2002; 29(12):798-805. doi:10.1097/00007435-200212000-00011

42. Bellis MA, Watson FL, Hughes $S$, et al. Comparative views of the public, sex workers, businesses and residents on establishing managed zones for prostitution: analysis of a consultation in Liverpool. Health Place. 2007; 13(3):60316. doi:10.1016/j.healthplace.2006.08.001

43. Boels D, Verhage A. Prostitution in the neighbourhood: Impact on residents and implications for municipal regulation. International Journal of Law, Crime and Justice. 2016; 46:43-56.

44. [author, blinded for review].

45. [author, blinded for review].

46. Weathers FW LB, Herman D, Huska J, Keane T. The PTSD checklist-civilian version (PCL-C). Boston, MA: National Center for PTSD; 1994.

47. Kroenke K, Spitzer RL, Williams JB. The PHQ-9: validity of a brief depression severity measure. J Gen Intern Med. 2001; 16(9):606-13.

48. Straus MA, Douglas EM. A short form of the Revised Conflict Tactics Scales, and typologies for severity and mutuality. Violence Vict. 2004; 19(5):507-20.

49. Erausquin JT, Reed E, Blankenship KM. Police-related experiences and HIV risk among female sex workers in Andhra Pradesh, India. J Infect Dis. 2011; 204 Suppl 5:S1223-8. doi:10.1093/infdis/jir539

50. [author, blinded for review].

51. Orchard T, Murie A, Elash H-L, et al. "People like us": spatialised notions of health, stigma, power and subjectivity among women in street sex work. Culture, health \& sexuality. 2019; 21(4):478-94.

52. Shannon K, Kerr T, Strathdee SA, Shoveller J, Montaner JS, Tyndall MW. Prevalence and structural correlates of gender based violence among a prospective cohort of female sex workers. BMJ. 2009; 339:b2939.

doi:10.1136/bmj.b2939

53. Roth AM, Kral AH, Mitchell A, Mukherjee R, Davidson P, Lankenau SE. Overdose prevention site acceptability among residents and businesses surrounding a proposed site in Philadelphia, USA. Journal of Urban Health. 2019; 96(3):341-52. 